\title{
sciendo
}

\author{
BULGARIAN ACADEMY OF SCIENCES
}

CYBERNETICS AND INFORMATION TECHNOLOGIES • Volume 21, No 1

Sofia $\bullet 2021$

Print ISSN: 1311-9702; Online ISSN: 1314-4081

DOI: $10.2478 /$ cait-2021-0006

\section{A Study on Human Learning Ability during Classification of Motion and Colour Visual Cues and Their Combination}

\author{
Albena Tchamova ${ }^{1}$, Jean Dezert ${ }^{2}$, Nadejda Bocheva ${ }^{3}$, Pavlina \\ Konstantinova ${ }^{4}$, Bilyana Genova ${ }^{3}$, Miroslava Stefanova ${ }^{3}$ \\ ${ }^{1}$ Institute of Information and Communication Technologies, Bulgarian Academy of Sciences, 1113 Sofia, \\ Bulgaria \\ ${ }^{2}$ The French Aerospace Lab, Palaiseau, France \\ ${ }^{3}$ Institute of Neurobiology, Bulgarian Acad. of Sci., Sofia, Bulgaria \\ ${ }^{4}$ European Polytechnical University Pernik, Bulgaria
}

E-mails: tchamova@bas.bg_jean.dezert@onera.fr_nbbocheva@hotmail.com pavlina.konstantinova@gmail.com b.genova@abv.bg mirad_st@abv.bg

Abstract: The paper presents a study on the human learning process during the classification of stimuli, defined by motion and color visual cues and their combination. Because the classification dimension and the features that define each category are uncertain, we model the learning curves using Bayesian inference and more precisely the Normalized Conjunctive Consensus rule, and also on the base of the more efficient probabilistic Proportional Conflict Redistribution rule No 5 (pPCR5) defined within Dezert-Smarandache Theory (DSmT) of plausible and paradoxical reasoning. Our goal is to study how these rules succeed to model consistently both: human individual and group behaviour during the learning of the associations between the stimuli and the responses in categorization tasks varying by the amount of relevant stimulus information. The effect of age on this process is also evaluated.

Keywords: Vision; Human Perception, Classification, Color cue, Motion cue, Cues Combination, DSmT, probabilistic Proportional Redistribution rule No 5 (pPCR5), Normalized Conjunctive Consensus (NCC) rule.

\section{Introduction}

In everyday activities, humans often have to classify objects and events in different categories. The process of classification requires the acquisition of the common characteristics of the members of a category. Depending on the category structure, three different ways are assumed to be employed in classification [1]: rule-based, incremental learning, or memorization of all exemplars. Rule-based classification is 
supposed to involve sequential hypothesis testing to uncover the rule of categorization. The incremental learning is supposed to be related to finding the category boundaries in cases when the stimulus categories are defined by more than one feature and no simple rule describes the category membership. It involves forming associations between a set of features and the responses. The third way to find the category assignments is by memorizing the associations and the responses for each combination of stimulus features.

When the stimuli for categorization are multidimensional and not all features are relevant for their classification, an important question is how humans find out the proper stimulus characteristics for category membership. To answer this question, [2] tested whether a normative strategy based on probabilistic inference could describe the process of category learning. Their modeling data imply that the decision making based on Bayesian inference is computationally too demanding and that humans use suboptimal strategies in the process of categorization.

In the present study, we use multidimensional visual stimuli that are divided into categories by rules of different complexity. The change in the rule of classifications changes in the amount of irrelevant information. We compare human cue combination performance in arbitrary (unstructured) classification task with modeled combination performance, based on particular fusion rules. In the presented study we apply and compare the performances of the following fusion rules: the Normalized Conjunctive Consensus (NCC), and the probabilistic Proportional Conflict Redistribution rule No 5 (pPCR5) defined within DSmT to model the human process of cue integration.

We focus on how the human age influences the process of classification as the experimental evidence implies that various brain structures and processes are involved in the different categorization tasks [3] and they do change differently with ageing [4].

This paper is organized as follows. In Section 2 we present briefly the principles of the used fusion rules, applied to model the human cue integration in a classification task. Section 3 is devoted to the experimental strategy, methods, procedures, stimulus, and subjects participating in the experiments. The results obtained are described and analysed in Section 4. In Section 5 fusion rules performance is presented and in Section 6 the trends are illustrated. Conclusions are made in Section 7.

\section{Fusion rules for modelling visual cue combination}

Various fusion rules exist in the literature to deal with uncertain data. They are based on different mathematical models and on different methods for transferring the conflicting mass onto the meaningful hypotheses about the problem under consideration. In this paper, we use the Normalized Conjunctive Consensus (NCC) rule and the probabilistic Proportional Conflict Redistribution rule no.5 (pPCR5), defined within Dezert-Smarandache Theory (DSmT) of Plausible and Paradoxical Reasoning [5]. Both these rules are described in detail in [6]. 


\section{Experiments}

Three experiments were performed.

\subsection{Stimuli}

The stimuli were dynamic patterns that differed by the motion direction, the spatial distribution, shape, and the color of the moving elements. Moving elements were either spheres or cubes. Two conditions were simulated - in one of them the elements were positioned on a plane, in the other they were randomly positioned in depth. The simulated motion could have 4 different directions: to the left, to the right, forward, or backward. As a result, eight different moving patterns were generated: a movement to the left among a cloud of elements, movement to the right among a cloud of elements, movement forward among a cloud of elements, movement backward among a cloud of elements, horizontal translation to the left, horizontal translation to the right, movement forward towards a plane, and movement backward from a plane. The moving elements - spheres or cubes, could have one of four colors: red, blue, green, or yellow. Of all possible combinations of movements, shape, and color of elements (64 in total: 8 movements $\times 4$ colors $\times 2$ shapes of moving elements) we randomly selected 16 combinations. The characteristics of the chosen stimuli are given in Table 1.

Table 1. Characteristics of the stimuli used in the study

\begin{tabular}{|c|c|c|c|c|}
\hline $\begin{array}{c}\text { Number of } \\
\text { stimuli }\end{array}$ & $\begin{array}{c}\text { Disposition } \\
\text { of elements }\end{array}$ & $\begin{array}{c}\text { Motion } \\
\text { direction }\end{array}$ & Color & $\begin{array}{c}\text { Shape of } \\
\text { elements }\end{array}$ \\
\hline 1 & cloud & forward & green & cube \\
\hline 2 & cloud & backward & yellow & sphere \\
\hline 3 & cloud & backward & green & cube \\
\hline 4 & cloud & backward & red & cube \\
\hline 5 & cloud & right & green & sphere \\
\hline 6 & cloud & right & yellow & cube \\
\hline 7 & cloud & left & blue & cube \\
\hline 8 & cloud & left & green & cube \\
\hline 9 & wall & forward & red & sphere \\
\hline 10 & wall & forward & yellow & cube \\
\hline 11 & wall & forward & blue & sphere \\
\hline 12 & wall & backward & blue & sphere \\
\hline 13 & wall & right & red & sphere \\
\hline 14 & wall & right & blue & sphere \\
\hline 15 & wall & left & yellow & sphere \\
\hline 16 & wall & left & red & cube \\
\hline
\end{tabular}

\subsection{Experimental conditions}

Three experiments were performed. They differed by the classification rule used to separate the stimuli into two categories. In Experiment 1 the stimuli were divided arbitrarily by the movement type that resulted from the disposition of the elements and the direction of motion, whereas the shape and the color of the elements were irrelevant. In Experiment 2, the stimuli were divided randomly into two categories 
based on their color, whereas the elements' spatial disposition, motion direction, and the shape of elements were irrelevant. In Experiment 3, the stimuli were randomly divided into two groups based on the combination of the motion direction, elements' disposition, and color. As in Experiments 1 and 2, the shape of the elements was irrelevant. Table 2 presents the separation of the elements in two categories for the three experiments.

Table 2. Characteristics of the stimuli used for dividing the stimuli in categories in Experiments 1-3

\begin{tabular}{|c|c|c|c|c|c|}
\hline Experiment 1 & Experiment 2 & \multicolumn{4}{|c|}{ Experiment 3} \\
\hline Category 1 & Category 2 & Category 1 & Category 2 & Category 1 & Category 2 \\
\hline cloud, right & cloud, left & red & blue & $\begin{array}{l}\text { cloud, right, } \\
\text { green }\end{array}$ & cloud, left, blue \\
\hline $\begin{array}{l}\text { cloud, } \\
\text { backward }\end{array}$ & $\begin{array}{l}\text { cloud, } \\
\text { forward }\end{array}$ & yellow & green & wall, left, red & $\begin{array}{l}\text { cloud, left, } \\
\text { green }\end{array}$ \\
\hline wall, right & wall, left & & & $\begin{array}{l}\text { wall, left, } \\
\text { yellow }\end{array}$ & $\begin{array}{l}\text { cloud, right, } \\
\text { yellow }\end{array}$ \\
\hline \multirow[t]{5}{*}{$\begin{array}{l}\text { wall, } \\
\text { backward }\end{array}$} & wall, forward & & & $\begin{array}{l}\text { wall, right, } \\
\text { red }\end{array}$ & $\begin{array}{l}\text { cloud, forward, } \\
\text { green }\end{array}$ \\
\hline & & & & $\begin{array}{l}\text { wall, right, } \\
\text { blue }\end{array}$ & $\begin{array}{l}\text { cloud, } \\
\text { backward, red }\end{array}$ \\
\hline & & & & $\begin{array}{l}\text { wall, forward, } \\
\text { red }\end{array}$ & $\begin{array}{l}\text { cloud, } \\
\text { backward, green }\end{array}$ \\
\hline & & & & $\begin{array}{l}\text { wall, forward, } \\
\text { blue }\end{array}$ & $\begin{array}{l}\text { cloud, } \\
\text { backward, } \\
\text { yellow }\end{array}$ \\
\hline & & & & $\begin{array}{l}\text { wall, forward, } \\
\text { yellow }\end{array}$ & $\begin{array}{l}\text { wall, backward, } \\
\text { blue }\end{array}$ \\
\hline
\end{tabular}

As is evident, the classification of the stimuli in Experiment 3 could be done either by trying to find the combination of the stimulus characteristics, or as a rulewith-exception as all cloud stimuli except one are in Category 2, and all wall stimuli except one are in Category 1.

\subsection{Experimental procedure}

Before each experiment, the calibration of an eye-movement recording device was performed. In addition to the standard calibration, each experimental session started with a sequential presentation of a dot at different positions (center, left, left corner, up, right corner, right, down) 10 degrees from the screen center. The dot changed position after $1.5 \mathrm{~s}$.

Each experiment started with the sequential presentation of all stimuli. At the end of the stimulus sequence, all stimuli were presented again in a different order and the Subject had to describe the stimulus characteristics - shape and color of elements, the direction of motion, and disposition of elements. This preliminary session aims to acquaint the subjects with the stimulus set and the stimulus characteristics. The experimental session consisted of 128 stimuli -8 repetitions of each stimulus in random order.

Before the presentation of each stimulus, a warning signal is given. The stimulus duration was $1 \mathrm{~s}$. During stimulus presentation, a fixation dot with a diameter of 0.5 degrees of arc was shown in the middle of the screen during stimulus presentation. 
Five hundred milliseconds after stimulus disappearance, two figures - a triangle and a star (with a size of approximately $4 \times 4$ degrees of arc) appeared at 10 degrees to the left or right from the fixation point. On every trial, the position of these figures was randomly selected. The Subject's task was to select to which stimuli corresponds the star, and to which - the triangle. They were required to keep fixation at the fixation point during stimulus presentation and to make a saccade to the selected figure. They had to press the left mouse button if the selected figure is to the left of the fixation point, and the right mouse button - if the selected figure was to the right. In the case of correct choice, a high tone was played, whereas in case of incorrect choice a low tone was played. The subjects were told that at the beginning they could only guess, but during the progression of the experiment, by trial-and-error, they would be able to find the proper classification of the stimuli in categories.

\subsection{Method}

The order of the experiments was contra-balanced between the subjects. The number of the experimental sessions depended on the Subject's performance - if the number of correct responses was low, the participants started a new session after a short break. However, even if the performance of a subject was still not good, no subject participated in more than three experimental sessions. The experiments were separated by 3 to 7 days to avoid inference from previously learned categorization.

\subsection{Apparatus}

The stimuli were presented on a black background with a custom program written in Python with OpenGl. They were presented on the computer screen operated in refresh rate $60 \mathrm{~Hz}$ and resolution 1280×1024 pixels, 21" Dell Trinitron with Nvidia Quadro 900XGL graphic board. The stimulus observation was binocular from a distance of $57 \mathrm{~cm}$.

The eye movements of the participants were recorded by Jazz novo eye tracking system (Ober Consulting Sp. Z o.o.) [7].

\subsection{Subjects}

17 young subjects, aged 18-38 years (median 22 years) and 17 elderly subjects, aged 63-75 years (median 67 years) took part in the study.

\section{Performance evaluation of age-related observers groups}

The experimental goal of our study is directed to characterize human decision making in a classification task influenced by:

- motion information only;

- color information only;

- combined motion and color information.

As the stimuli were randomly assigned to different categories based on their visual characteristics, the test Subjects have to find the correct association between 
the stimuli and the outcome by trial-and-error. While in the classification of objects or events in categories a generalization of their characteristics is needed, in arbitrary categorization a specific representation of the stimuli is required. As the stimuli in all experiments were the same, one possibility is that irrespective of the categorization rule, the participants would represent them in working memory by all cues. In this case, their performance would be similar in all conditions and the memory load would be equivalent. Also, if unstructured categorization is based on procedural memory [8], the number of features used to categorize the stimuli would be irrelevant. However, the experiments in our study could be also characterized as rule-based with rules of varying complexity that change the amount of irrelevant information. A more efficient way to classify the stimuli is to represent them in memory only by the cues determined by the categorization rule ignoring the irrelevant stimulus characteristics.

An example of the performance from the experiments of an occasional test person is shown in Fig. 1. It represents the proportion of correct responses in blocks of 16 trials. This information is processed and analyzed to get conclusions about the characteristics used for classification in different categories.

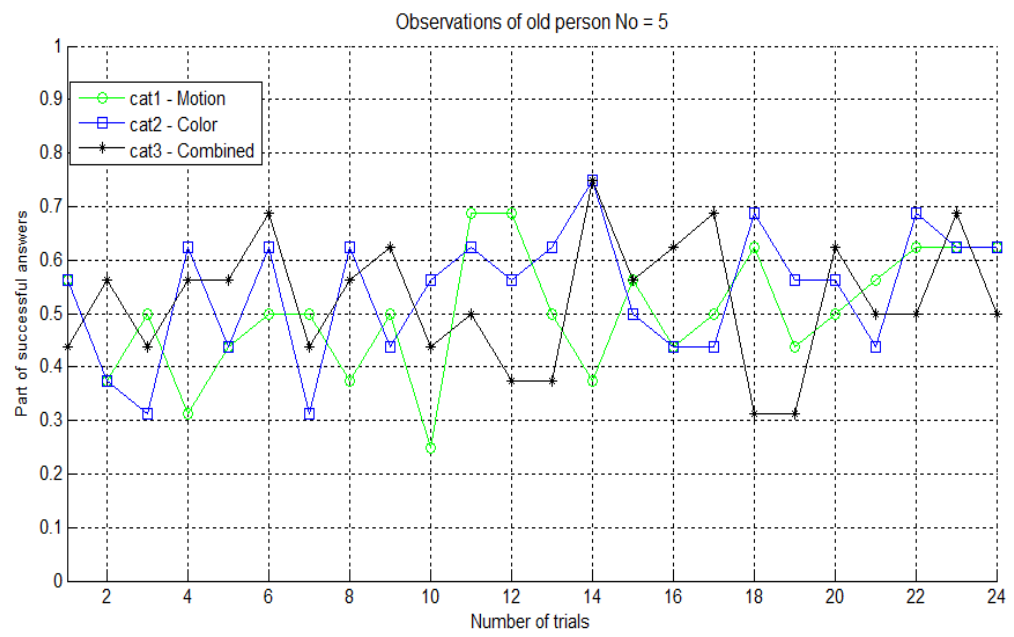

Fig. 1. Observations from an occasional test person

The question is whether the people rely and base their responses on a single source of information, or on a combined one, and also which type of information utilized is more informative in the decision process and corresponds best to the rule used for separating the stimuli in categories. The participants belong to two age groups: Young and Old. Hence, also the influence of human age on the assessment of the decision will be evaluated. The evaluation is made on the base of experimental learning curves, obtained for all different experimental categories and for each subject in all age-contingent groups. The learning curve represents the change in the correct responses with some measure of the experience gained, i.e., the number of trials. 


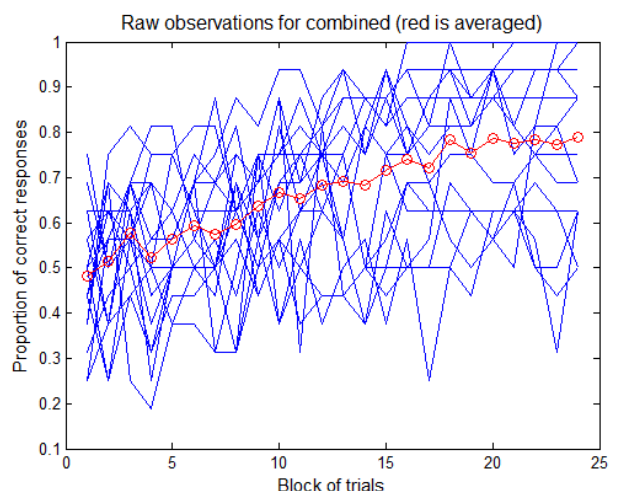

Fig. 2. Raw observations (blue), averaged over subjects (red)

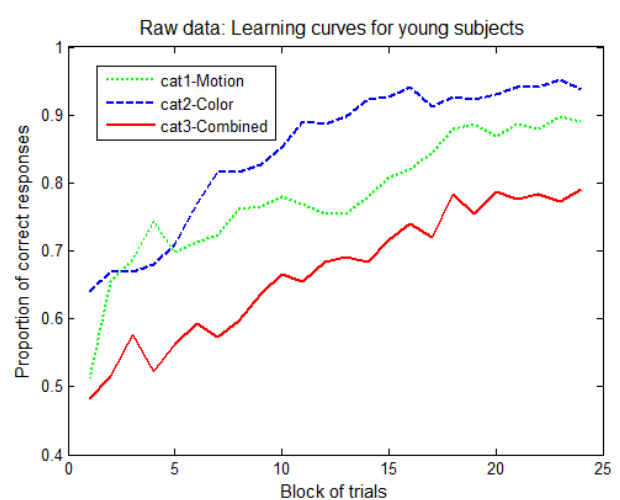

Fig. 3. Experimental learning curves for the three categorization rules

Fig. 2 presents the learning curves of all young subjects in the case when the stimuli were divided arbitrarily into two groups based on the combination of the color and motion of the stimulus elements. The figure clearly shows the large individual differences in task performance. It also demonstrates that with the increase of blocks, the performance of the group improves. Fig. 3 represents the averaged learning curves for all subjects in the young group. It implies that the performance gradually increases and the rate of increase is different for the different categorization rules.

\subsection{Evaluation of the perception in Young observers group}

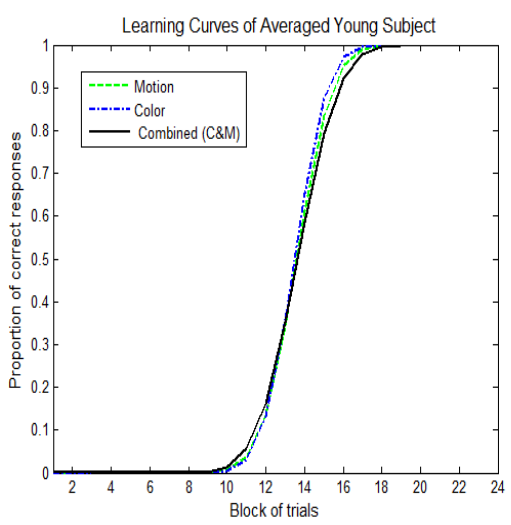

Fig. 4. Learning curves of the averaged young subject

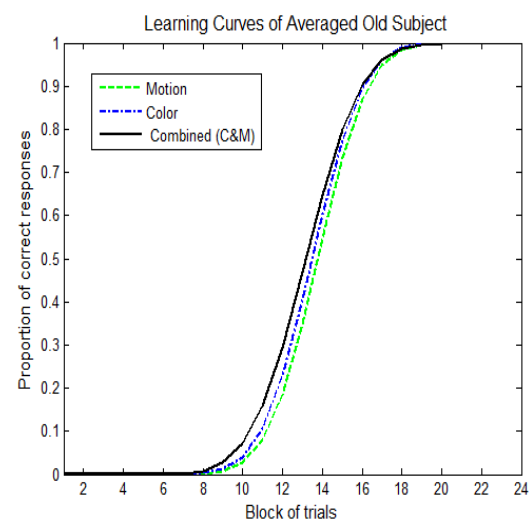

Fig. 5. Learning curves of an averaged old subject

The comparison of the performance in the motion and color conditions show that in the Young group only six out of 17 observers have the best performance for the motion condition, nine observers effectively utilized the color information showing the best performance in this case, and only one observer shows best performance in the combined condition (for two other observers the performance in 
the combined condition is equivalent to that in a single-cue case). For one observer the learning performance is equivalent to both single-cue conditions. The cumulative curve representing the distribution of the average correct responses (on the base of 17 subjects in the group) of the young subjects is shown in Fig. 4.

For the averaged young subject the learning curves associated with motion, color, and combined information are not distant.

\subsection{Evaluation of the perception in Old observers group}

The comparison of the performance in the motion and color conditions shows that in the Old group 9 out of 17 observers have the best performance for the motion condition, six observers show better performance using the color information. For two out of 17 observers the learning curves for both motion and color information could be considered as equivalent. The performance of averaged Old test person on the base of 17 subjects in the group is shown in Fig. 5. Here again, the best performance of the single-cue category is confirmed though the difference between the three conditions is not significant.

It can be summarized that the participants in each group learn best the association between the stimuli and the response when the categorization rule is based on a single cue. This implies that the memory representation of the stimulus characteristics is determined by the categorization rule and the participants are able to ignore the stimulus features irrelevant to the category membership.

It is interesting to compare young and old test persons for the same conditions. We put together the learning curves of young and old subjects in Fig. 6 for the categories motion and color and category combined in Fig. 7.

The results show that for both single- and multiple-cue categorization rules the older participant perform worse than the younger one, especially for the case of the combination of color and motion information where the difference between the two groups seems greater.

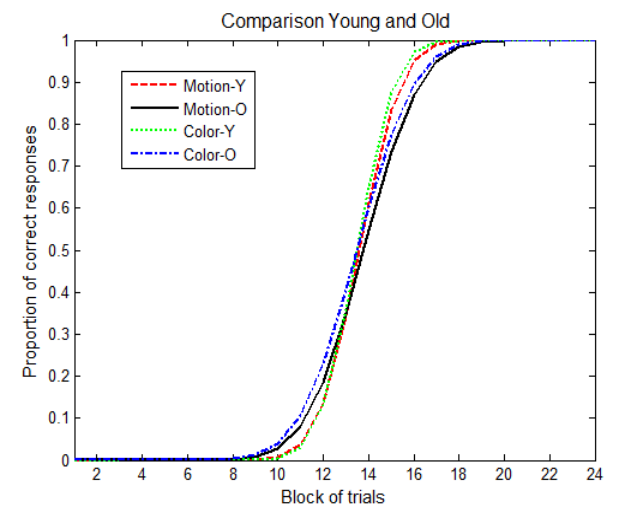

Fig. 6. Learning curves of Young and Old for Color and Motion categories

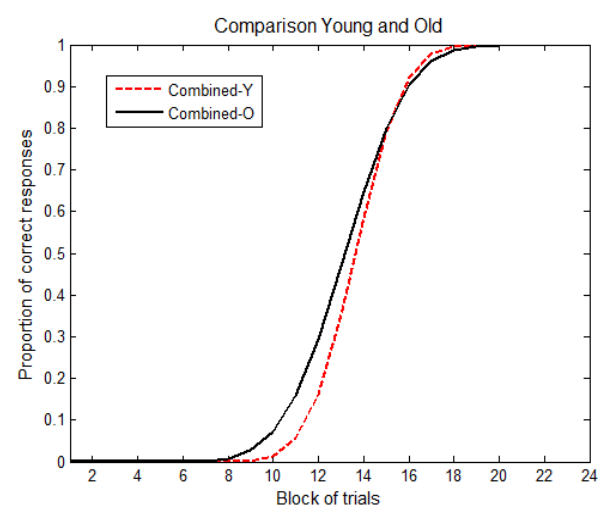

Fig. 7. Learning curves of Young and Old for Combined category 


\section{5. pPCR5 and NCC rules performance for predicting human's way of motion and color combination in decision making}

The main question here is which fusion rule - pPCR5 or NCC used to combine available motion and color information predicts more adequately human cue integration in deciding the stimulus category?

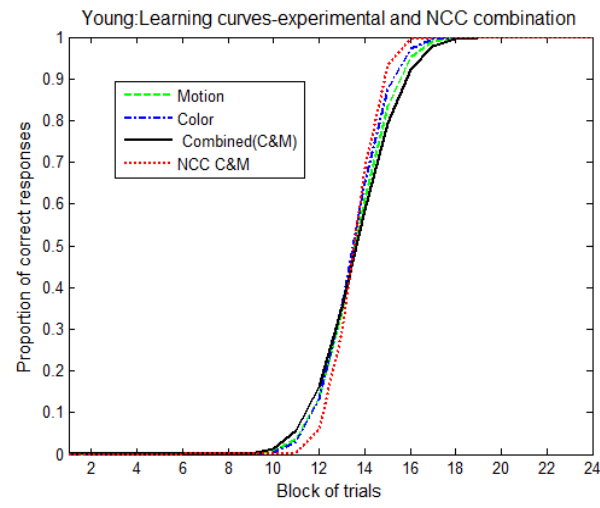

Fig. 8. Learning curves for experimental categories and mathematically obtained NCC result for the averaged young subject

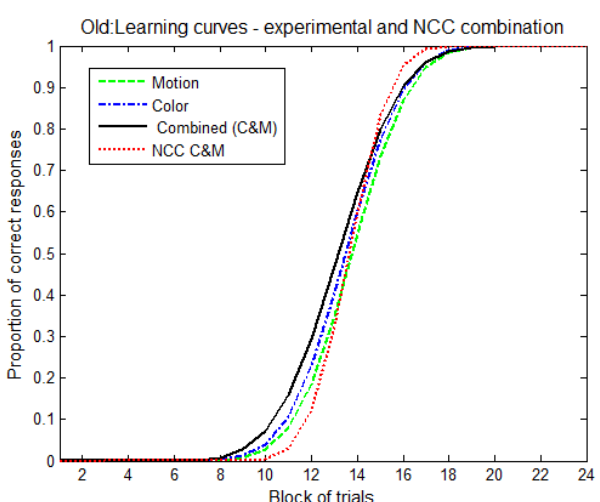

Fig. 9. Learning curves for experimental categories and mathematically obtained NCC result for the averaged old subject

In order to answer this question, we need to make a comparison between experimentally obtained and predicted (via pPCR5 and NCC rules) learning curves for combined categories (motion and color), for the two age groups.

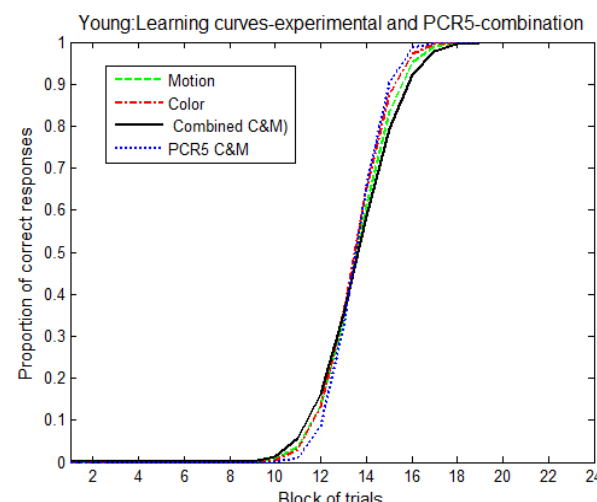

Fig. 10. Learning curves for experimental categories and mathematically obtained PCR5 for result for the averaged young subject

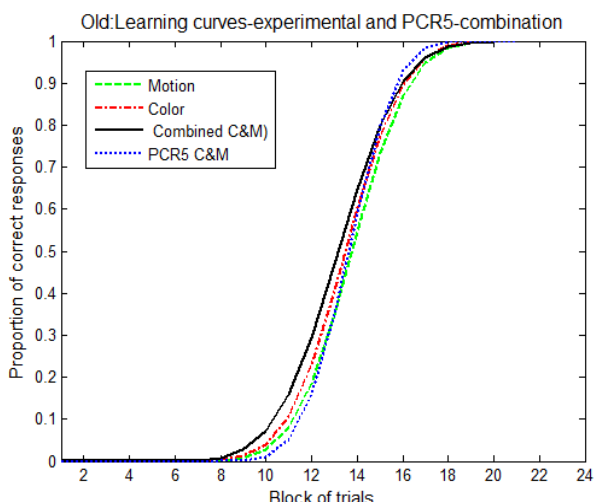

Fig. 11. Learning curves for experimental categories and mathematically obtained PCR5 result for the averaged old subject 
In Figs 8 and 9, are shown the results of mathematical modeling by NCC, and in Figs 10 and 11 results of applying pPCR5 are presented.

In Figs 12 and 13 the comparison of the empirical and both mathematical methods are given.

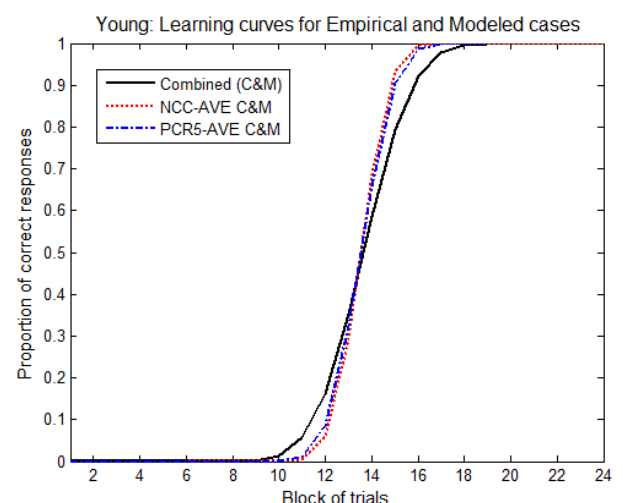

Fig. 12. Learning curves for empirical and mathematically modelled cases for young

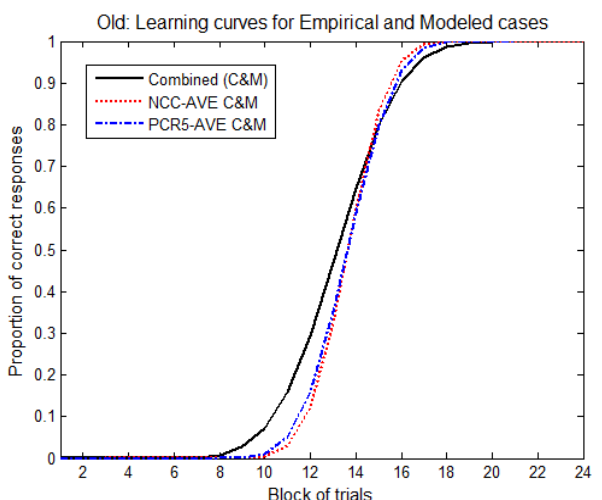

Fig. 13. Learning curves for empirical and mathematically modelled cases for old

The results of the mathematical modeling based on both rules predict better performance than observed in the experimental data. This conclusion concerns the averaged learning curves for the two groups. However, due to the large individual differences in each group, the average learning curve might not be representative of group performance. In Section 6 we present a different approach to describe the learning in the two groups and apply the same mathematical modeling to it.

Another comparison between the methods is provided on the base of the goodness-of-fit test [9], which is an important application of chi-squared criteria:

$$
\chi^{2}=\sum_{j=1}^{J} \frac{\left(O_{j}-E_{j}\right)^{2}}{E_{j}},
$$

where $\chi^{2}$ is an index of the agreement between an Observed $(O) /$ /experimental and Expected $(E) /$ predicted via particular fusion rule sample values of the learning curve. For our case, $J=24$ represents the number of independent observations. The critical value of the test for $v=J-1=23$ degrees of freedom at the assumed probability $p=0.1$ is $\chi^{2}=32.0$ [9].

The respective results are given in Table 3 for the young group, in Table 4 - for the old persons' group.

In general, the results show that both fusion rules- NCC and pPCR5 succeed to predict adequately human performance for the two age groups. Only for one subject from Table 4, the NCC modeling is not adequate - because its NCC error is bigger than the defined critical value $\chi^{2}=32.0$. Thus, contrary to the case of the average learning curves where the NCC and pPCR5 predict better performance than obtained experimentally, both rules can describe well the individual learning curves in both age groups. 
The results for young and old test-persons are presented in Tables 3 and 4, respectively.

Table 3. Chi-squared values for young subjects

\begin{tabular}{|c|c|c|}
\hline Subject & pPCR5 (Motion and Color) & NCC (Motion and Color) \\
\hline 1 & 25.4366 & 0.4469 \\
2 & 0.0747 & 0.5870 \\
3 & 0.8255 & 6.4822 \\
4 & 0.5054 & 1.2087 \\
5 & 28.2272 & 1.0270 \\
6 & 1.2497 & 3.6587 \\
7 & 23.9470 & 1.4417 \\
8 & 0.3935 & 0.3370 \\
9 & 8.5851 & 0.3969 \\
10 & 0.5281 & 3.1269 \\
11 & 16.0195 & 3.7373 \\
12 & 14.5225 & 9.4381 \\
13 & 1.0158 & 0.0756 \\
14 & 11.4884 & 0.3826 \\
15 & 22.0764 & 0.3815 \\
16 & 17.7547 & 1.1310 \\
17 & 28.0757 & 0.2587 \\
\hline
\end{tabular}

Table 4. Chi-squared values for old subjects

\begin{tabular}{|c|c|c|}
\hline Subject & pPCR5 (Motion and Color) & NCC (Motion and Color) \\
\hline 1 & 0.3587 & 1.7369 \\
2 & $* * * * * *$ & $* * * * * *$ \\
3 & 0.7524 & 0.9697 \\
4 & 13.1982 & 14.2576 \\
5 & 6.2634 & 11.0246 \\
6 & 8.0005 & 11.0022 \\
7 & 0.9172 & 3.3941 \\
8 & 0.0441 & 0.0923 \\
9 & 10.0382 & 9.8545 \\
10 & 0.0471 & 0.2213 \\
11 & 11.3722 & 15.2725 \\
12 & 2.3003 & 38.7541 \\
13 & 2.4354 & 31.1429 \\
14 & 24.7161 & 9.5761 \\
15 & $* * * * * *$ & $* * * * *$ \\
16 & 0.8704 & 9.2390 \\
17 & 6.5457 & 21.1361 \\
\hline
\end{tabular}

****** means missing information from the test person.

\section{Common trends of age-related observer groups}

The goal here is to find the common trend, concerning the performance of the two groups. For this purpose, we consider each group as a set of different sources of evidence, associated with each person in the group. That way the young group consists of 17 (young subjects) sources of evidence, which should be combined all together via pPCR5 and NCC fusion rules. 
The combined individual behaviours in a particular group are estimated, revealing its intrinsic behaviour as a whole, reducing uncertainties associated with individual performances. All tested subjects in the age groups are considered as independent and equally reliable sources of information because each subject provides his/her learning curve, associated with the motion and color condition and should be taken into account with equal weights to derive these trends.

Our goal is to find out which combinational rule (pPCR5 or NCC) is able to model correctly and adequately such human age-contingent group trends in the process of decision making. The results obtained for experimental and estimated (via the fusion rules) trends, concerning the cues combination groups' performance are presented in Figs 14 and 15. It can be seen that the learning curves obtained by the pPCR5 fusion rule in the two figures - for young and old test persons, are more close to the experimentally defined target curve.

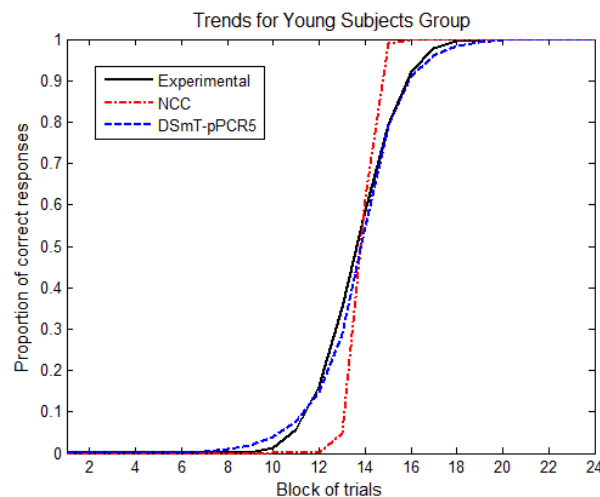

Fig. 14. Trends for Young subjects group

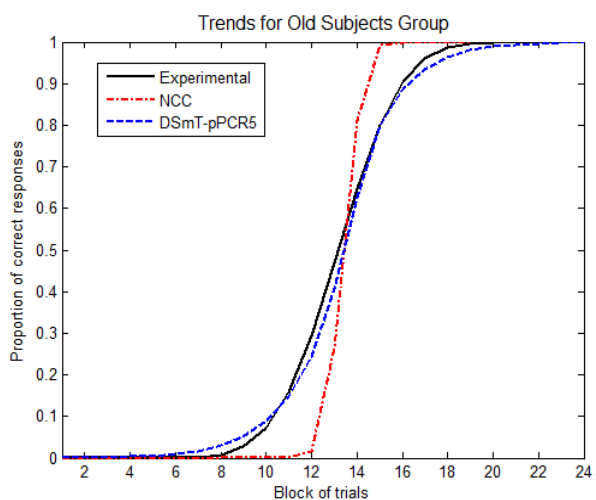

Fig. 15. Trends for Old subjects group

pPCR5 fusion rule predicts more correctly the human model of decision making, than the NCC rule, utilizing all the available information (Motion and Color), even in case of conflict. NCC based trends are very sensitive to the sources (different subjects' learning curves) with the bigger means, neglecting that way part of the available information and acting as an amplifier of the information by reducing the variances

\section{Discussion}

This paper presents a study on the human classification of stimuli defined by motion and color visual cues and their combination. The influence of human age on this process is evaluated. The results obtained show age-related differences in the performance of the subjects in estimating the human classification based on both single- and multi-cue classification rules.

Our experimentally obtained data for young observers suggest a smaller effect of the motion information, while for the older observers the color information has 
less effect. Hence, the learning performance differs depending on the categorization rule and the age of the participants. All age-related groups have difficulties to divide the stimuli in groups based on combined (motion and color) information. This finding implies that this condition presents a greater memory load to all observers than the single cue conditions.

In the classification task of multi-cue stimuli, there is uncertainty about which dimension and which feature along this dimension determine the correct response. Hence, the observers have to determine not only the classification rule that specifies the categorization dimension, but also which exemplars that differ by this dimension fall in one or the other category. In contrast to the previous studies $[2,10,11]$ testing Bayesian inference in classification studies and learning, we do not model the explicit performance of each subject or group based on the available cues. Instead, our approach reminds the analysis of cue combination in perception studies where the proportion of correct responses is related to stimulus strength. Thus, in our analyses, the experience gained during the task performance is regarded similarly to stimulus characteristics in detection or discrimination perceptual tasks. We perform a comparison between experimentally obtained and predicted (via pPCR5 and NCC rules) learning curves for combined condition (motion and color), for the two age groups and apply the goodness-of-fit test, one important application of chi-squared criteria, to evaluate the correspondence of the experimental and the model data. The results suggest that the predictions of the models outperform human performance for both age groups. This finding differs from our previous results [6] on cue combination in evaluating the heading direction from texture and motion cues. However, it coincides with the conclusion in [2] that human subjects perform suboptimally in categorization tasks.

Both the NCC and the pPCR5 rules predict well the individual learning curves with a slight advantage of the pPCR5 rule as it fits well all the learning curves. This finding provides evidence for the relevance of our approach for analysis of the learning curves.

We evaluate the common trend in the performance of the two age groups by considering each group member as an independent source of information. The obtained trends are better described by the pPCR5 rule than by the NCC rule. The best fit of the group behavior by the PCR5 rule is due to its properties to utilize all available information even in a case of conflict between the individual learning curves. It is an appropriate characteristic of the group data as it preserves the idiosyncrasies in the performance of each individual and hence, represents effectively the process of decision making in classification tasks for different age groups.

Acknowledgments: The reported work is a part of and was supported by the Project DN02/3/2016 'Modelling of voluntary saccadic eye movements during decision making' funded by the Bulgarian Science Fund. 


\section{References}

1. A s h b y, F. G., V. V. V a l e n t i n. Multiple Systems of Perceptual Category Learning: Theory and Cognitive Tests. - In: Handbook of Categorization in Cognitive Science. 2nd Ed. H. Cohen, C. Lefebvre, Eds. New York, Elsevier, 2017, pp. 157-188.

2. Wils on, R. C., Y. Niv. Inferring Relevance in a Changing World. - Frontiers in Human Neuroscience, Vol. 5, 2012, 189.

https://doi.org/10.3389/fnhum.2011.00189

3. Shohamy, D., C. E. My ers, J. Kal a n ith i, M. A. Gluck. Basal Ganglia and Dopamine Contributions to Probabilistic Category Learning. - Neuroscience and Biobehavioral Reviews, Vol. 32, 2008, No 2, pp. 219-236. https://doi.org/10.1016/j.neubiorev.2007.07.008

4. K a l r a, P. B., J. G a b r i e li, A. S. F i n n. Evidence of Stable Individual Differences in Implicit Learning. - Cognition, Vol. 190, 2019, pp. 199-211. https://doi.org/10.1016/j.cognition.2019.05.007

5. F. Smarandache, J. Dezert, Ed. Advances and Applications of DSmT for Information Fusion. American Research Press, Vols. 1-4, 2004-2015. https://www.onera.fr/staff/jean-dezert/references

6. Tchamova, A., J. Dezert, P. Konstantinova, N. Bocheva, B. Genova, M. S t e f a n o v a. Human Heading Perception Based on Form and Motion Combination. - In: Proc. of IEEE International Conference on INnovations in Intelligent SysTems and Applications (INISTA'18), 3-5 July 2018.

7. http://www.ober-consulting.com/9/lang/1

8. S e g e r, C. A., C. M. C in c o t t a. The Roles of the Caudate Nucleus in Human Classification Learning. - J. Neurosci, Vol. 25, 2005, pp. 2941-2951.

DOI:10.1523/JNEUROSCI.3401-04.2005.

9. M a tr e, J., G. G i 1 b r e a th. Statistics for Business and Economics. 3rd Edition. 1987.

10. Y u, A. J., P. D a y a n. Uncertainty, Neuromodulation, and Attention. - Neuron, Vol. 46, 2005, pp. 681-692. DOI: 10.1016/j.neuron.2005.04.026.

11. Gers h m a n, S. J., J. D. Coh e n, Y. N i v. Learning to Selectively Attend. - In: Proc. of 32nd Annual Conference of the Cognitive Science Society, Portland, 2010.

Received: 14.10.2020; Accepted: 11.01.2021 